\title{
Detectors for LGS WF sensing on an ELT: characteristics, special features, limitations, technology
}

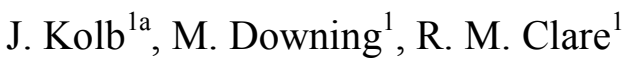 \\ ${ }^{1}$ European Southern Observatory, Karl-Schwarzschild-Strasse 2, D-85748 Garching bei München, \\ Germany
}

\begin{abstract}
The wave front sensing of Laser Guide Star (LGS) light with a Shack-Hartmann sensor (SHWFS) on an ELT requires a large fast low-noise visible detector with high Quantum Efficiency (QE), low latency, low charge diffusion and dark current, and excellent cosmetic quality. Such a device doesn't exist yet, although the CCD technology is already stretched to its limits in order to deliver detectors for the soon-to-come next generation of AO systems (e2v CCD220 for the VLT AO Facility and SPHERE). For three years ESO has been pushing the development of a technological solution that satisfies the need of the AO systems for the European ELT (E-ELT) project. This paper presents the requirements on the detector as well as how they originated and describes the development plan followed by ESO in order to find a suitable technological solution.
\end{abstract}

\section{AO need for detectors}

The E-ELT is a project of 42-meter aperture optical telescope designed by ESO which is currently in phase B (detailed design). It will be an adaptive telescope, i.e. Adaptive Optics will be full part of the telescope design, including a deformable mirror in the optical train, and the continuous use of (GL)AO to drive the telescope while improving at the same time the site seeing conditions. In addition, instruments are being designed (at a phase A level) that will provide various modes of postfocal AO correction. As part of the WFS, and thus being one of the three main components of an AO system together with the DM and the RTC, the detectors required by AO on the E-ELT fall into one of the four following categories:

1) Infrared detectors, for Tip-Tilt or low-order IR WFS. Current developments are ongoing at ESO in the field of $\mathrm{HgCdTe}$ eAPDs arrays $(256 \times 256)$ with $\mathrm{RON}<5 \mathrm{e}-\mathrm{rms}$ and no excess noise.

2) Large visible fast low-noise detector for Shack-Hartmann based AO WFS on NGS (GLAO and SCAO) and LGS (GL-, LT-, MC- and MO- AO) are the focus of the present paper.

3) $3 \mathrm{kHz}$ low-noise detector for XAO.

4) Existing visible high performance detector (e.g. e2v's CCD220) that could be used for guiding, Tip-Tilt visible sensors, "first-light" low-order AO, and also pyramid, SH quad-cell or other similar WFS.

Although the CCD technology is pushed to its limits in order to deliver detectors for the soon-tocome next generation of AO systems (e2v CCD220 for the VLT AO Facility and SPHERE), the requirements of the E-ELT AO systems are far from being met. In order to fill the gap, ESO started in 2006 to fund the development of large visible fast low-noise WFS detector, as detailed in $\S 3$. In the long term, this work aims on at the production of 30 to 50 fully operational detectors that could

\footnotetext{
a e-mail : jkolb@eso.org
} 
be used in all the E-ELT AO WFS. The requirements for this device are driven by the most complex AO systems (LGS-assisted LTAO and MCAO) but would also be used in the simpler LGS GLAO and NGS GL- and SC-AO systems. This strategy is intended to reduce costs by minimizing the number of new developments to one, and maximizing the number of units to be produced (i.e. to reduce recurrent costs).

In the case of a 42-meter diameter ELT and the side launching of the LGS, the SHWFS of the intended MCAO system requires a 1680 x 1680 pixels detector that should be able to run up to 700 $\mathrm{Hz}$ and deliver images with a Read-Out Noise (RON) better than 3 electrons per pixel and per frame. In addition to those, high QE, low latency, charge diffusion and dark current and excellent cosmetic quality are mandatory. The origin of some of the requirements will be developed in $\S 2$. The detector development led by ESO is described in $§ 3$.

\section{Detector requirements}

The justification of the requirements is often the result of simulations performed by Richard Clare [1], as seen in all the plots in the section. Note that those so-called "open-loop" simulations aim at providing comparative results between different values of a specification, and do not provide absolute WFE values for any particular AO system, as they don't take into account the whole AO loop, in particular the temporal behavior.

\subsection{Spatial resolution}

The requirement for linear actuator spacing on the quaternary mirror (M4) of the E-ELT is half a meter, i.e. 85 actuators across the telescope diameter (with a goal of 127), thus the detector is required to cover $84 \times 84$ sub-apertures, and the technology should allow an extension to $126 \times 126$.

AO simulations (see Fig. 1, left) show that at least $14 \times 14$ pixels per sub-aperture are required in order to cover spot elongation in the most demanding AO systems. In order to account for the largest FWHM of the sodium layer, the requirement was set to $20 \times 20$ pixels. The same simulations give an optimum pixel number of 6x6 pixels for NGS WF sensing (with no elongation). Those results lead to respectively 1680x1680 and 504x504 pixels for the NGS and LGS detectors.

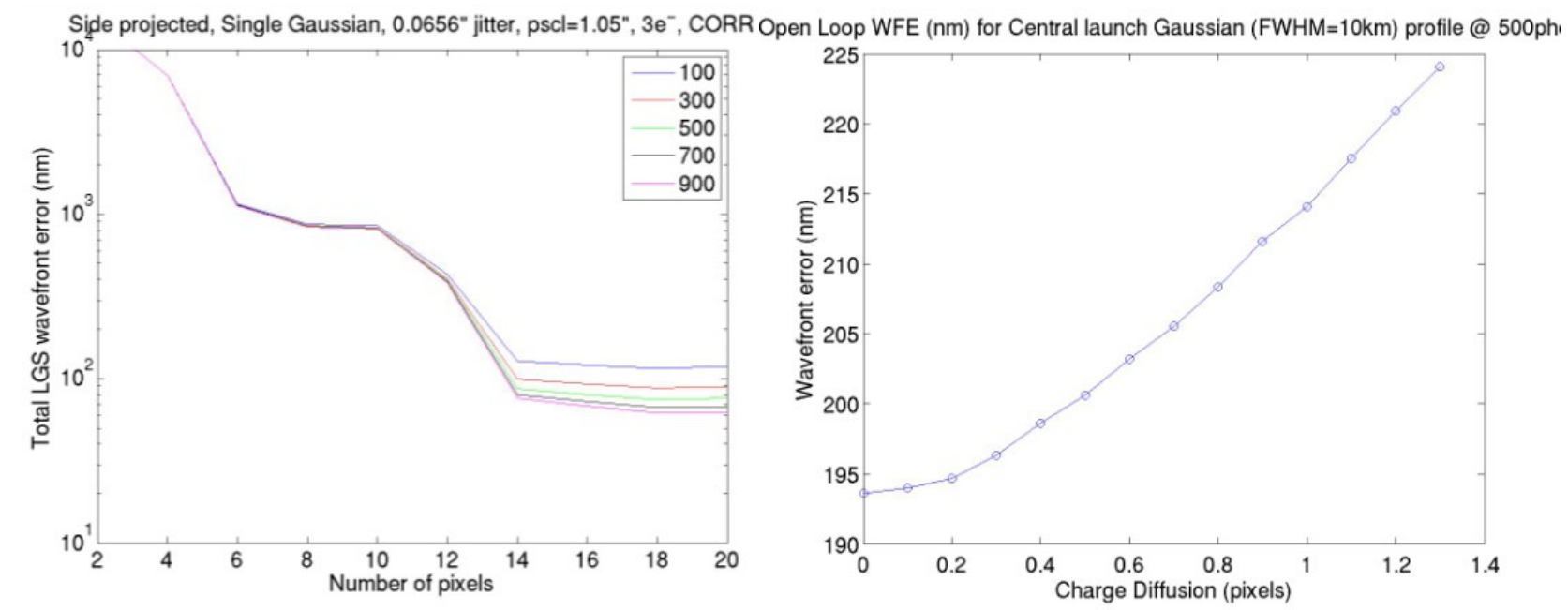

Fig. 1. Left: Wavefront error as a function of the linear number of pixels per sub-aperture, for different number of photons per sub-aperture and per frame (top to bottom: 100, 300, 500, 700 and 900). Right: Wavefront error as a function of the pixel PSF. 
Another requirement linked to spatial resolution is the charge diffusion or detector PSF. It is the FWHM of the detected spot when the light beam is of a smaller dimension than the pixel and hits it in the center. It comes from the fact that photo-electrons diffuse in the detector material before being detected. If a $2 \mathrm{k} \times 2 \mathrm{k}$ detector has a PSF of 2 pixels, its spatial resolution is not better than that of a $1 \mathrm{k} \mathrm{x} 1 \mathrm{k}$ detector with 1 pixel PSF. For our application this value was specified to be better than 0.8 of a pixel, which is about half the expected LGS spot size in the non-elongated dimension (see Fig. 1 , right).

Finally the size of a pixel is required to be in the range from 20 to 28 microns (largest detector size of about $\sim 40 \times 40 \mathrm{~mm}$ ), which is a compromise between a reasonable sized detector (smaller detectors have a higher yield) and the easiness of alignment (bigger pixels are better).

\subsection{Temporal resolution}

A good temporal resolution is essential when one aims at correcting in "real-time" the atmospheric turbulence. As the Real-Time computer and the DM, the WFS detector is required to have a fast response. In practice it translates into several requirements:

1) Short exposure time: simulations carried out by the E-ELT instrument consortia show that exposures from 1.4 to $10 \mathrm{~ms}$ are required, corresponding to frame rates from 100 to $700 \mathrm{~Hz}$.

2) Non-synchronicity with a sub-aperture: should be smaller than $20 \mu \mathrm{s}$, as some detector architectures require pixels to be read-out at different times, which is not desired if ones wants to have a "snapshot" of the sub-aperture spot.

3) Latency: the read-out and data transfer should be fast $(<$ half the exposure time), and continuous, so that the RTC is able to compute centroids in a pipe-line fashion.

4) Image lag = "charges left behind": smaller than 2\% per pixel at the next frame. This defect is equivalent to a reduction of the $\mathrm{AO}$ bandwidth.

In addition, the full-well capacity of the pixels was required to be larger than 4000 electrons, a compromise between the detection of large signals and of smaller signals with enough accuracy.

\subsection{Image quality}

One of the most important requirements is the one on detector noise, mainly Read-Out Noise (RON) which is required to be smaller than 3 electrons per pixel and per frame. In this range, simulations show that having one electron less of RON allows a saving of around $30 \%$ on the required laser power (see Fig. 2)!

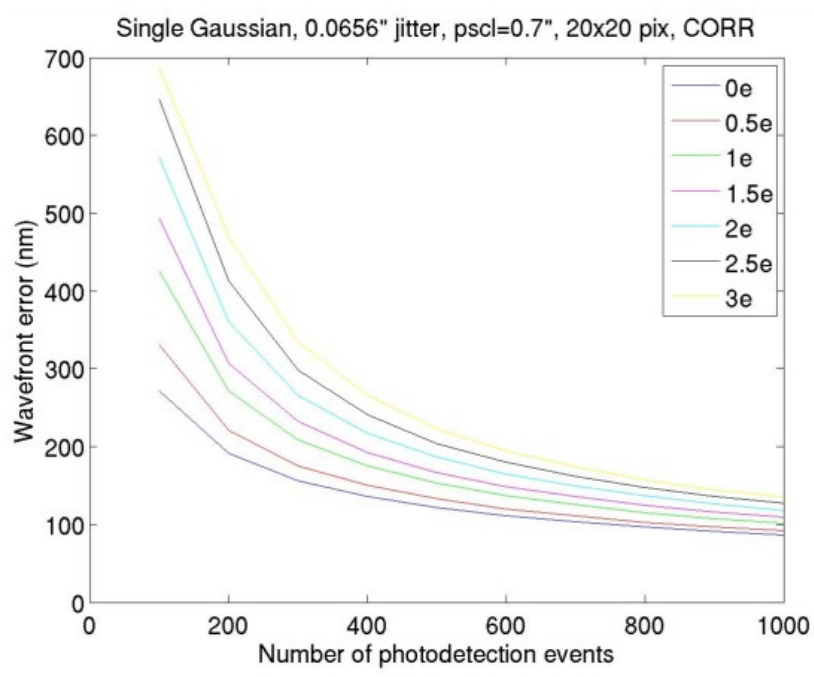

Fig. 2. Wavefront error as a function of the number of photons per sub-aperture per frame, for different values of detector Read-Out noise (from bottom to top: 0 to 3 with steps of 0.5 electrons per pixel and per frame). 
Another noise source, especially at low frame rates, is dark current which is required to be smaller than 0.5 electron per pixel and per frame. It is interesting to notice that in the case of some technologies, the RON and dark current are not the same for all pixels (see Fig. 2) [2], and that the effect of those deviations is noticeable on the WFE (see Fig. 4), so it is necessary to have the limit that more than $95 \%$ of the pixels have less than twice the mean RON/Dark current.
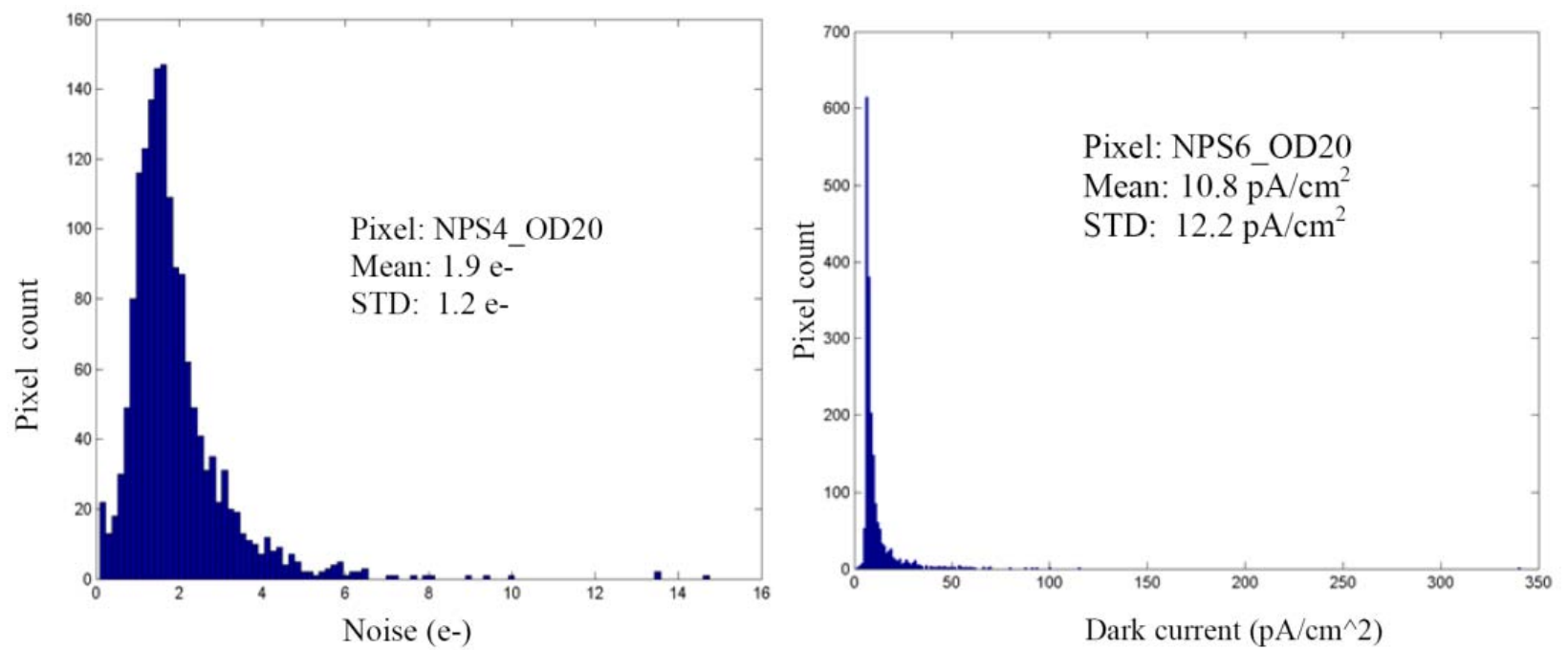

Fig. 3. Example of the distribution of RON (left) and dark current (right) in a CMOS pixel (from [2]).
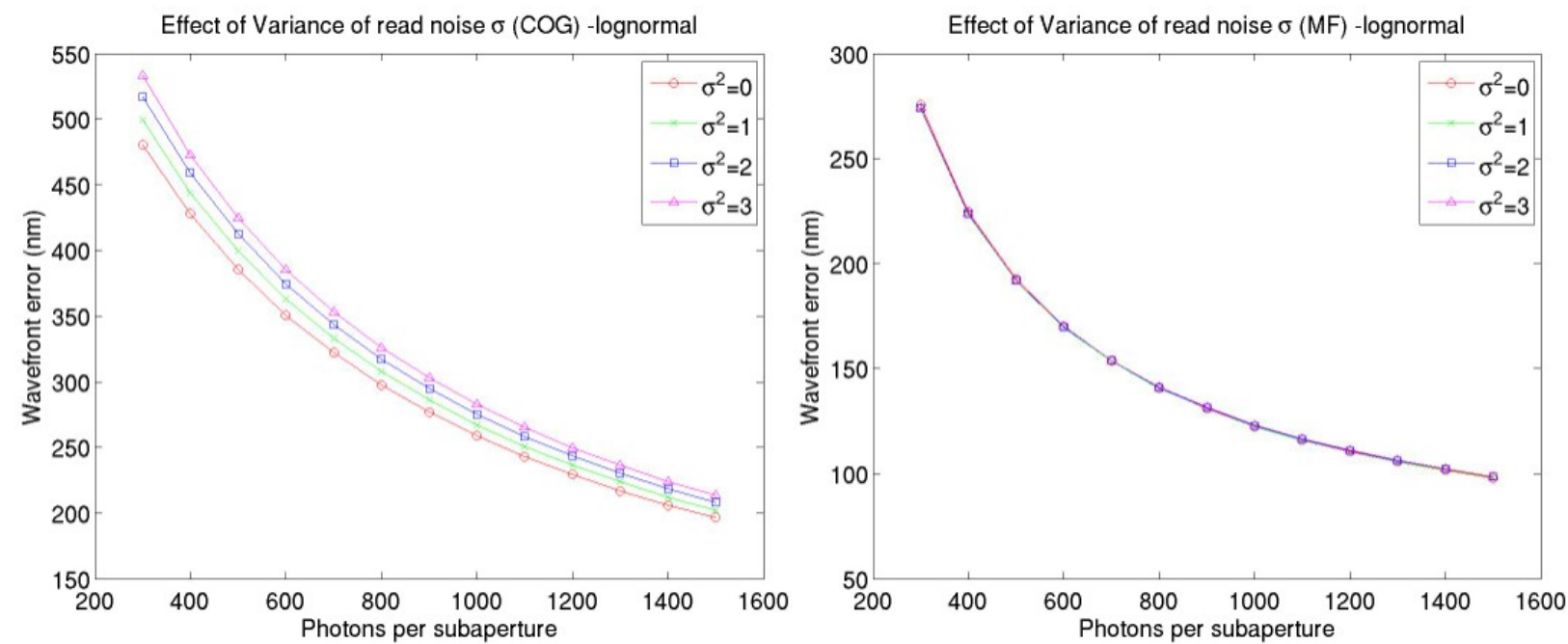

Fig. 4. Effect of the variance of RON on the WFE as a function of the number of photons per sub-aperture and per frame. On the example of a mean RON of 3 electrons per pixel per frame, the cases shown are from bottom to top: $\sigma^{2}=0,1,2$ and 3 electrons ${ }^{2}$. Left: using CoG algorithm for centroiding. Right: Match Filter.

Finally, the detector response to light (Quantum Efficiency QE) should be maximized. The required minimum and goal values for the E-ELT LGS WFS detector are listed in the Table 1.

Table 1. Minimum and goal requirements on $\mathrm{QE}$ at different wavelengths.

\begin{tabular}{|l|r|r|r|r|r|}
\hline Wavelength $(\mathrm{nm})$ & 500 & 600 & 700 & 800 & 900 \\
\hline Minimum requirement & $85 \%$ & $85 \%$ & $65 \%$ & $35 \%$ & $10 \%$ \\
\hline Goal requirement & $95 \%$ & $95 \%$ & $95 \%$ & $80 \%$ & $50 \%$ \\
\hline
\end{tabular}




\subsection{Other requirements}

In addition to the requirements listed in the three paragraphs above, there are several others that put constrains on the design of a possible detector suitable for our application: linearity, packaging, incidence angle, interfaces, pixel response non uniformity, cosmetics, stability with temperature and time, lifetime...

\section{Technological solutions}

The plan for the development of the E-ELT LGS WFS detector is as follows:

- Feasibility studies (Mar-Dec '07): four contracts (two of which were funded by the FP6 ELTDS and another two by the E-ELT Phase A). Those studies investigated many different technologies, and concluded that the most promising ones were CMOS, APD array and orthogonal EMCCD.

- Technology Demonstrator phase (Jul '08 - Oct '09): three contracts (two of which were funded by the FP6 ELT-DS and one by the E-ELT Phase B). The goal was to retire pixel risk by demonstrating low noise and high speed with good imaging capability. Those studies succeeded in achieving the goal, but discovered additional problems to be investigated before implementing the pixels in an array.

- Scaled-Down Demonstrator phase (Mar '10 - Jun '12): one contract (half of which will be funded by the OPTICON FP7 JRA2 and half by the E-ELT Phase B). During this phase the selected contractor will retire architectural risks by designing and building a version of the E-ELT LGS WFS detector that meets all the requirements except for the size and the packaging. It should be possible to use this device as a NGS WFS detector on the E-ELT.

- Full-Size Demonstrator phase (2013 - 2014): The goal of this phase is to scale up the NGS detector, and should be only an engineering exercise.

- Production (2015 - 2016): 30 to 50 devices will be produced to equip the E-ELT.

A possible scheme of the final full-size detector is shown on the Fig. 5, where one can also see the sections addressed in the frame of the Technology Demonstrator and Scaled-Down Demonstrator phases.

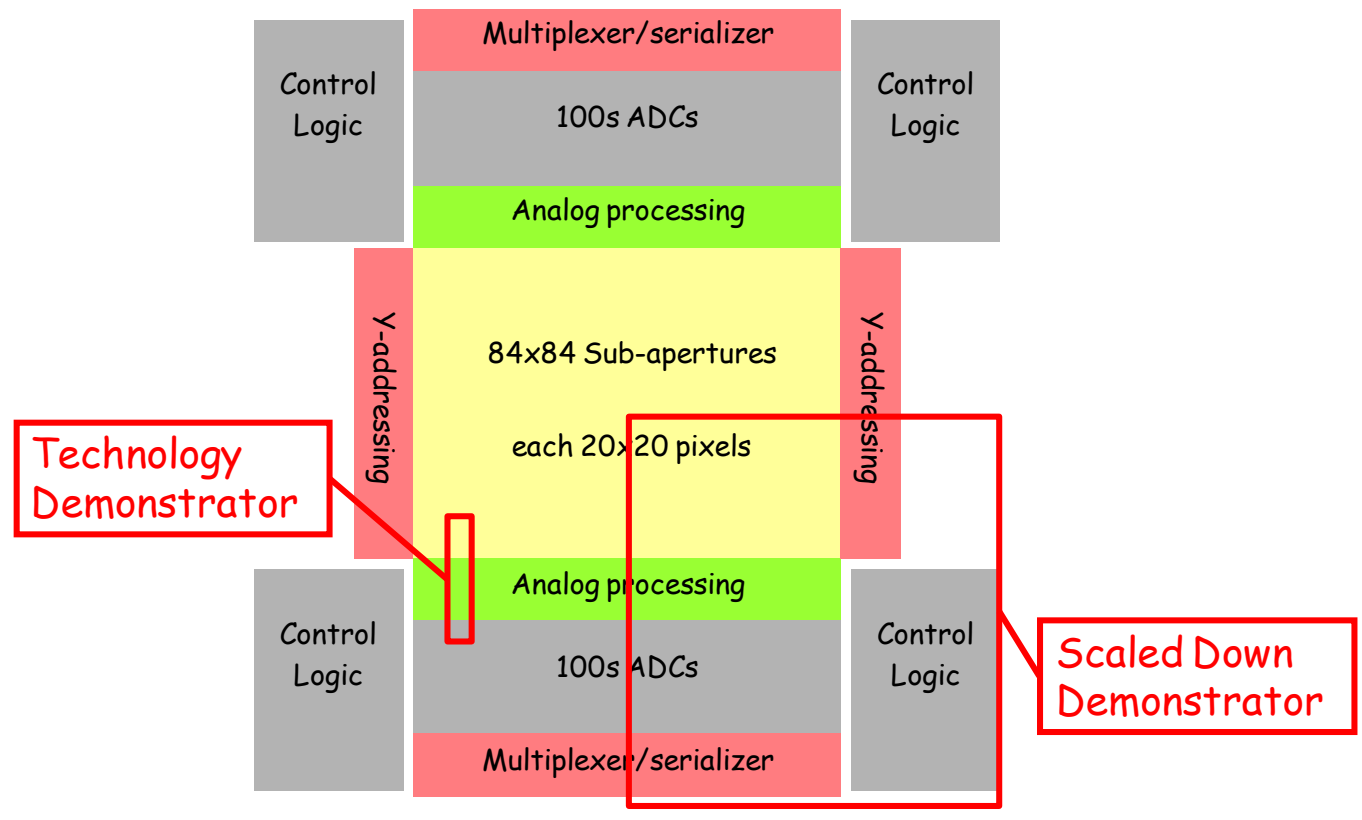

Fig. 5. Possible E-ELT AO WFS detector design. 


\section{Development status and conclusion}

At the time of writing this paper, the Technology Demonstrator phase is coming to an end, and the conclusion is that the unique combination of high speed, large number of pixels, low noise, and large pixels is a challenge for the design of the pixel and the detector architecture. Even though QE, RON, image lag, and high speed have been demonstrated in other devices, it had never been all done in the same chip before the Technology Demonstrator.

2010 will see the first step of the SDD phase, which will be done in parallel with the conceptual design of a controller for the E-ELT LGS WFS detector performed by the Laboratoire d'Astrophysique de Marseille (France) in the framework of the FP7 OPTICON JRA2 activities funded by the European Community.

\section{References}

1. R. M. Clare, M. le Louarn, S. Oberti, A. Garcia-Rissmann, Adaptive optics simulations for the European Extremely Large Telescope, in Adaptive Optics systems, Proc. SPIE 7015 (2008)

2. C. Liu, B. Fowler, H. Do, S. Mims, D. Laxson, B. Frymire, High Performance CMOS Image Sensor for Low Light Imaging, Proc. 2007 Int. Image Sensor Workshop, pp.327-330 (June 2007) 